\title{
REMOTE VIEWING OF CONCEALED TARGET PICTURES UNDER LIGHT AND DARK CONDITIONS
}

\author{
Stanley Krippner ${ }^{1}$, David T. Saunders ${ }^{2}$, Angel Morgan ${ }^{1} \&$ Alan Quan ${ }^{3}$ \\ ${ }^{1}$ Saybrook University, CA, USA \\ ${ }^{2}$ The University of Northampton, Northampton, UK \\ ${ }^{3}$ California State University, Long Beach, CA, USA
}

\author{
Corresponding Author Details: \\ Dr David T. Saunders \\ david.saunders@northampton.ac.uk \\ The University of Northampton \\ Psychology Division \\ Faculty of Health and Society \\ Park Campus, Fawsley Building \\ Northampton \\ Northamptonshire \\ England \\ NN2 7AL
}

\section{Keywords: Remote Viewing; RV; Free-response; Target Numinosity; Numinosity; Dark; Light}

\begin{abstract}
HeAding)
Context

The belief that darkness plays a facilitating role in remote viewing and other psi-related phenomena is well established in esoteric and traditional beliefs (Grim, 1983; Hallowell, 1942; Lyon, 2012). However, the role of darkness in RV success is unclear beyond these esoteric explanations.

Objective

This study explored the differential effect of darkness/light on remote viewing ability alongside the effect of time and their potential interaction.

Design

From an initial sample of twenty, seven remote viewers contributed a total of nineteen sessions each (nine light/ ten dark) which utilised randomized target selection, free-response descriptions, and ratings by both participants and an independent judge.

$\underline{\text { Results }}$

The usable data gave the edge to dark condition performance; the difference was not statistically significant. However, the study suffered from significant participant attrition, with only 7 participants completing the full number of trials. On the whole, participants who left the study early reported they did not find the target pictures "engaging," "interesting," or "emotionally involving". Schwartz (2007) has discussed the importance of targets being imbued with numinosity, or the perceived ability of a target to evoke an emotional state in the participant. Therefore exploratory post-hoc analyses concerning the numinosity of target images were conducted, to determine if this characteristic was associated with success. For the numinosity ratings of target images, a mean difference of $11.24,95 \%$ CI $[.12,22.3]$ was shown as significant, with the target images of participant 'hit' sessions containing higher numinosity ratings than unsuccessful 'miss' sessions $(t(11.47)=2.22, p(2$-tailed $)=$ $.048)$ with a large effect size $(d=1.02,[.01,1.99])$.
\end{abstract}




\section{Conclusion}

The findings may have implications for the use of participant judgement in future remote viewing research. Furthermore, because there are several advantages to what parapsychologists refer to as "free response" targets as opposed to "forced choice" targets (Honorton, 1975), the findings for target numinosity may have implications for the future selection of target material.

\section{INTRODUCTION}

Remote viewing (RV) has been defined by Stephan Schwartz as "a series of nonlocal consciousness formalized protocols" where a person provides "detailed information about a person, place, object, or event which information they should not be able to know by reason of their being shielded from it by time, space, or both" (Schwartz, 2017).

The term "nonlocal consciousness" has been used to describe this phenomenon; one demonstrated to have odds of occuring by chance at a billion to one (Tressoldi, 2011). For context Schwartz highlights that the Higgs-Boson was hypothesised to exist based on a one in 300 million chance that it did not exist (Schwartz, 2014).

Non-local consciousness has received mention throughout recorded history and has been used by nation-states for strategic purposes for millennia. The Egyptians, the Greeks, the Etruscans, and the Romans all maintained institutions with the purpose and function of creating a group of what today we would call remote viewers. In ancient times when oracles were honored, their task was to provide practical guidance based on the results of their attempted remote viewing (Myers, 1880; Inglis, 1977).

The oldest known recorded experiment in remote viewing is described in the $46^{\text {th }}$ chapter of The History of Herodotus, written by the Greek Herodotus of Halicarnassus, arguably the world's first historian, in 440 BCE. Herodotus recounted how King Croesus of Lydia carried out the first experiment in what today we would call anomalous perception (or ESP, extra-sensory perception). Croesus had received news that Lydia was going to be attacked by the Persians. He wanted to consult an oracle to tell him what to do but was not sure which one he could trust. The solution he came up with was the first description of what we know of today as remote viewing. He sent couriers to all the famous oracles of his day and gave each messenger the same task. They were to count the days from the time they left Lydia, and on the hundredth day, they were to ask the oracles what Croesus, King of Lydia, was doing at that moment. The 
answers were to be taken down in writing and delivered back to him. Only the most accurate description has survived, one from the oracle at Delphi. On the $100^{\text {th }}$ day, the oracle at Delphi answered in this hexameter verse:

But the number of sand I know, and the measure of drops in the ocean;

The dumb man I understand, and I hear the speech of the speechless:

And there hath come to my soul the smell of a strong-shelled tortoise

Boiling in caldron of bronze, and the flesh of lamb mingled with it;

Under it bronze is laid, it hath bronze as a clothing upon it.

(Schwartz, 2016, p. 405)

The Lydian courier wrote this down and faithfully reported back to Croesus. Of all the oracles, the Delphi Oracle was the only accurate description because Croesus had, on that $100^{\text {th }}$ day, cut both a tortoise and a lamb into pieces with his own hands. He had boiled them in a cauldron with a lid, both made of brass. In stipulating 100 days, with both the messenger and the oracle unaware of Croesus' activity on that day, Croesus had set up an experiment that is very similar to the blinding protocols used by researchers in remote viewing studies today (Schwartz, 2016). King Croesus' experiment was double-blind because neither the couriers nor the oracles knew the real purpose of the experiment nor what the king would be doing on the $100^{\text {th }}$ day.

\section{Darkness and Remote Viewing}

Throughout history, putative psi phenomena were thought to be fostered by conditions of darkness. The use of darkness in remote viewing and other psi-related phenomena is well established in esoteric and traditional beliefs (Lyon, 2012); however, the role of darkness in RV success is unclear outside of these esoteric explanations. Certain traditional healing practices, such as the Sioux yuwipi and the shaking tent ceremonies during which advice of their spirits are sought, are performed in the dark (Grim, 1983; Hallowell, 1942; Lyon, 2012). 
Early physical mediums required darkness or near darkness for the production of their phenomena, it was claimed that light, particularly short wavelength blue light was particularly damaging to the delicate ectoplasmic strucutres produced by the mediums (Gauld, 1982) and is sill emphaised by many contemporary physical mediumship groups. The Scole Group, for example performed only in the dark on the grounds that this condition works better, or they were so directed by their spirit guides (Keen, Ellison, \& Fontana, 1999), or in the cases of claimant mediums Kai MuEgge and David Thompson, because the introduction of light would cause the withdrawal of so-called "ectoplasm" and evoke possible injury to the medium. Certain ethnic groups hold funeral services at night to facilitate a safe transition of the purported "spirit" of the deceased (Laughlin, 2011).

These are but a few examples of the potential efficacy of darkness in connection with reported psi from explanations by esoteric and/or traditional practices.

Assuming non-local consciousness is achievable as previous studies have demonstrated, the modalities underlying it are not currently understood. In some cases of remote viewing, telepathy may be the means by which distant information is acquired when an agent is at the target location who is experiencing what the remote viewer attempts to describe. In other cases where there is no agent to perceive the target location or image, some form of clairvoyance or precognition may be at work. Another explanation may be that the remote viewer can have an out-of-body experience (OBE), which enables them to somehow extend their consciousness to the location of the target site or image and view it directly (Targ \& Puthoff, 2005).

In one of the earliest RV experiments, Targ and Puthoff tested the ability of a single claimant remote viewer by having one of the experimenters go to a randomly selected location while the participant remained in the laboratory, and the participant was asked to describe the location. In subsequent variations of these experiments, a set of geographical coordinates was supplied to the participants, who then attempted to describe the scene at those coordinates. The participants produced drawings and descriptions of the remote locations that were judged to be highly accurate and far beyond what would be 
expected by chance alone, without any apparent way of knowing that information through ordinary sensory means (Targ \& Puthoff, 1976).

More recent studies have used static photographs and dynamic video sequences as targets. For example, Lantz, Luke, and May (1994) tested the effect of static target images on remote viewing ability compared with dynamic target images. The level of correct RV identification of targets was statistically significant for both static and dynamic images. Furthermore, the presence of an agent (a telepathic sender) viewing the images made no difference in successful target identification, which appears to demonstrate that telepathy is not required for successful RV.

Regarding the accuracy and reliability of RV data over the years, across the three well-known RV labs: SRI, MOBIUS, and PEAR, "about 75 percent of what could be objectively evaluated proved to be correct" (Schwartz, 2017). In an evaluation of RV, statistician Jessica Utts (1995) stated, "The statistical results of the studies examined are far beyond what is expected by chance." Even renowned critic Ray Hyman (1995) replied in agreement to Utts that certain RV effect sizes reported were "too large and consistent to be dismissed as statistical flukes" (p.44).

Given the relative success of previous RV research and the requirement to understand the underlying modalities which may be conducive to RV success the aims for this study concerned an exploratory investigation into the potential differential effect of darkness or light on purported RV ability, the accuracy of RV ability over time, and their potential interaction.

\section{METHODS}

In this study, a total of 20 sessions were randomly ordered for a total of 10 light and 10 dark. All participants were requested to take part in each of the sessions. A factorial design was used to explore the differential effect of darkness/light on purported remote viewing ability. The effect of time (across the 20 sessions) and the interaction of time with light or dark conditions were also considered in relation to the number of successful target hits reported. 
The analysis of this study was submitted as an exploratory investigation for the study pre-register of the Koestler Parapsychology Unit (KPU) of the University of Edinburgh, and was approved following several revisions. The submitted documents principle focus was the planned procedures for statistical analysis, as data collection had already occured by this stage, however nothing had been sent to the second author, as an external researcher, for statistical analysis. The plans were confirmed and stored in the KPU's online data base. This procedure ensured full transparency of the planned analyses and inhibited any form of data "fishing" or "fudging," from using alternative evaluative methods which might favor the desired outcome. As an important aside, it is intended that the sixth hypothesis included within the KPU registration regarding geomagnetic correlates will be presented in a future paper.

\section{$\underline{\text { Participants }}$}

Quota sampling was used to recruit 20 remote viewer claimants (13 female/7 male). Most participants were recruited through the Energy Medicine University in Sausalito, California, where they were either faculty members or had been advanced RV students. Other participants were known by the authors to have been involved in RV activities and / or classes; thus all participants had prior RV experience. These participants were tested across a total of 20 sessions designed by Experimenter A, 10 of the sessions were arranged to take place with the participants in the light condition, and 10 in the dark condition. Randomized target selection, free-response descriptions, and ratings by both participants and an outside evaluator (i.e., independent judge) were utilized.

Materials

Images were full-color prints taken from slide images prepared by the parapsychologist Charles Honorton in the 1970s (Honorton, 1975). Images were drawn from a pool of 484 such slides by Experimenters $\mathrm{C}$ and $\mathrm{D}$. Of these, 50 pairs of images were randomly selected, through tossing dice, so that each image in the sets of 50 pairs would be used only once. An additional tossing of dice was sometimes neceessary to ensure that the subject matter of the two images of each pair differed from each other in order to make judging easier. Each image was placed in a single sealed envelope by Experimenter C. Each image had a unique numeric identifier written on the envelope exterior as well as 
on the back of each image. The envelopes were labelled with unique numbers ( 1 through 50$)$ to allow for random selection of image pairs during the experiment. This number also appeared on the back of each image, e.g., 1A, 1B, 35A, 35B. The alphanumeric coding of the pairs of images represented the pairs (i.e., "A" or "B" but did not designate target or decoy labelling; the numeric represented the pair). The numeric identifiers used for both the individual image envelopes and the pair of envelopes had no relationship to the appearance or content of the images thus identified. The envelopes had tamper-evident seals so that if anyone attempted to open an envelope before the end of any session, that activity would have been revealed by a broken seal. All envelopes were kept in the office of Experimenter B.

\section{Procedure}

Before each session, Experimenter B randomly selected one pair of images from the 50 image pairs, using a random number table. Experimenter B flipped a coin to determine which of the two images of the pair would serve as the target image for the session. The other image of the pair would be made the decoy image for that session. The numeric identifiers of both the target image and the decoy image were recorded and associated with each specific session.

After the random selection for each session, Experimenter B placed it on a table; the image remained sealed in its envelope and unseen by Experimenter B. The sealed target image was located in the same room at Experimenter B's home for each session throughout the study. The decoy image was left in Experimenter B's office and was placed on a shelf separate from, but in the same room as the rest of the decoy images.

Before the beginning of each session, Experimenter A sent the latitude and longitude coordinates of the location of the target image to the participants via e-mail, which they were instructed to use or ignore as they saw fit. Participants were told that the target would be an image enclosed in a sealed envelope that had been placed on a table at the given coordinates. The participants were also informed whether each session was to be in the light or dark condition. Participants were told that the envelope containing the image for each session would remain in this position for 48 hours. Experimenter B drew subsequent pairs from the remaining pool of images and repeated the same process for each session. 
Since there were initially 50 pairs of images, and only 40 to be used, random selection of pairs was preserved throughout the experiment. If a pair were mistakenly selected more than once, that session would be counted as a legitimate session, however this scenario never occured. If the randomly selected decoy target from the pair was incorrectly placed on the table that session would not be used and a makeup session would be scheduled; this occured once during the course of the study. If Experimenter B did not select the pairs as dictated by Experimenter A, that session would not be used; this occured once throughout the course of the study. Participants were informed that any target could be used again in one or more subsequent sessions. This was done to prevent participant bias against subject matter of targets after a target was seen due to the belief that the same subject could not appear again.

A total of 20 remote viewing sessions was planned with each participant expected to participate in all 20 sessions. Ten sessions took place with the participants in the dark condition and ten in the light condition. While the targets' images were located in the same room throughout the study, the room was brightly lit during the light condition and was dimmed (with lights off and shades drawn) during the dark condition. Participants were instructed to dim the lights in the room they were located during the dark condition and to brighten their room during the light condition, with participants requested to use the same room for all sessions if possible. This was to maintain a consistent remote viewing environment and rule to avoid differences in the room size or configuration as a potential confounding variable, causing differences in performance between the light and dark conditions.

All participants received blindfolds (sleeping masks) of the same type, and the participants performed remote viewing while wearing these in both light and dark conditions, providing participants with a uniform visual field in all sessions. It was hoped that this would rule out increased concentration ability as a factor in determining performance. Without the blindfolds, the dark condition would provide less visual stimuli than the light condition, and thus any higher scoring during the dark condition could be attributed simply to increased concentration rather than to the darkness of the environment. Due to some participants finding the blindfolds cumbersome, it was decided to make the blindfolds use optional rather than compulsory for participants. The researchers requested that paticipants record their usage of the 
blindfolds so that future comparions could be made, however several participants failed to conform to this request making meaningful comparisons impossible.

To ensure that each participant understood the procedure, each remote viewer participated in a pilot session. A pair of targets was sent to Experimenter B, selected for this session only with the same pair used for all participants. This pilot session was a light session, with all following sessions randomly determined. The general protocol for the pilot session was adhered to for all future sessions.

One day before each session, by flipping a coin Experimenter A randomly determined whether the session would be dark or light with the provision that half of the sessions would be dark and half light. To ensure this division, randomization was not used for the final three sessions of the study. The participants were informed whether the session would be dark or light a day before the session was to begin. This allowed participants time to prepare for remote viewing and to set up their environment to create the required dark or light environment.

Experimenter B recorded the date and started time of the session. The participants had been instructed by Experimenter A to remote view the image at a time that was convenient for them during the designated 48 hour period. They remote viewed for about 30 minutes in the condition that had been specified for each session and recorded the date and start/end times of their remote viewing period.

For each session, participants were asked to handwrite or type (by typewriter or computer) verbal free-responses (i.e., those not restricted in content or format) on paper, describing their impressions of the target image. Participants recorded these impressions after they removed their blindfolds at the end of their remote viewing period. They were also asked to hand-draw a depiction of the target image on paper. During the dark condition, participants were allowed to use a small light source, such as a small incandescent flashlight or single candle flame, to provide enough light for them to see what they were recording on paper. Participants sent Experimenter A their handwritten impressions and sketches via postal mail (or by email attachment if they had access to computer scanning).

After each participant sent their descriptions to experimenter A, Experimenter A then emailed digitized images of both the target image and the decoy image to the remote viewer to score each image 
regarding the correspondence to the image they remotely viewed. They were not informed at this point which image was the target or decoy image. The participants were asked to rate each of the two images on a scale of " 1 " (meaning absolute certainty the image was not the target) to "100" (meaning absolute certainty the image corresponds to the image they remotely viewed). They were requested to avoid tied scores.

Once completed, participants sent their ratings of both images to Experimenter A for recording. After all ratings from all participants were received by Experimenter A via email and all handwritten impressions from all participants had been received by Experimenter A by postal mail, Experimenter B informed Experimenter A of the coded identity of the correct target. At this point, Experimenter A sent out a digitized image of the correct target for the session to all participants to provide feedback to the participants regarding their performance for that specific session. Following the pilot testing of the protocol two sessions occurred each week throughout the study, i.e., Mondays and Thursdays.

\section{Independent Judge Evaluation}

The targets developed by Honorton (1975) made a binary evaluation possible, based on the presence or absence of 10 content variables. In this way, the participant's response could be compared to both the actual target and the control target. This procedure, although commendable, was not used in this study because participants did not respond uniformly. Instead, the participants and the independent judge were asked to check responses for correspondences to two targets (the actual and the decoy) on a 100point scale that had been used effectively in the Maimonides Dream Laboratory experiments (e.g., Krippner, Honorton, \& Ullman, 1971). The independent judge had a background in both dream studies and filmmaking, hence was quite familiar with the type of images emanating from this study. She submitted the following description of her procedure:

In preparation to serve as the independent judge for this study, Experimenter D and I went over the 100-point scoring system I would be using, which had been utilized in past studies conducted at the Maimonides Dream Laboratory. We conducted a practice session and went through the 
rating process. I was requested to avoid tied scores. After all of the participant, self-ratings had been completed; Experimenter A emailed to me digitized images of both the target image and the decoy image for one session at a time to judge (labelled simply with Session \#, Image A and Image B). I was not informed at this point which image was the target or decoy image. I rated each image regarding its correspondence to the notes from each participant's RV session, which Experimenter A emailed to me along with the images. Based on each of the participants' RV notes, I rated each of the two images on a scale of " 1 " (meaning absolute certainty the image was not the target) to "100" (meaning absolute certainty the image corresponded to the image they remotely viewed). Once each judging session was completed, I sent my ratings for both images by all participants, to Experimenter A. After he received all of my ratings for all sessions, Experimenter A informed me which images (A or B) had been the targets, and which had been the decoys for each session.

\section{Research Hypotheses}

The experimental hypotheses under consideration are as follows:

Hypothesis One: There will be a significant mean difference in target hits taken from the average of independent judge target-decoy difference values of all sequential trials as analyzed by single sample ttest.

Hypothesis Two: There will be a significant mean difference between remote viewers' selfcorrespondence target image scores and the independent judge's correspondence raw scores across all sequential trials as analyzed by single sample t-test.

Hypothesis Three: There will be a significant mean difference between remote viewers' selfcorrespondence target image scores and the independent judge's correspondence raw scores when comparing light vs dark sessions as analyzed by an independent group t-test. 
Hypothesis Four: There will be a significant main effect for the number of hits taken from independent judge target-decoy difference values when comparing light vs dark sessions as analysed by an independent group t-test.

Hypothesis Five: There will be a significant interaction effect in the number of hits, taken from independent judge target-decoy difference values between sequential trial and light vs. dark sessions as analyzed by a mixed factorial ANOVA.

\section{RESULTS}

Due to technical issues in session three where it was noticed the wrong target envelope had been pulled, this session's data were discarded leaving a total of 19 sessions (10 in the dark condition and 9 in the light condition). Furthermore, participant attrition was a significant factor in this study which requires mention. In total only seven participants completed all 19 sessions, substantially impacting the study's statistical power. This may also suggest an optional stopping problem, as participants were provided feedback on a session by session basis they may have dropped out when performing poorly. While a follow up, which shall be discussed shortly with those that dropped out suggested against this reason as their primary motivation all results must be viewed with this caveat in mind.

Table 1 demonstrates the descriptive statistics for the seven remote-viewers who participated in each of these 19 judging sessions, with scores for these sessions shown for both the remote-viewers and the independent judge. The descriptive statistics show a tendency for the independent judge to successfully determine the correct target more often than the remote viewers. 
Table 1: Descriptive statistics for session per Remote Viewer, recorded by Remote Viewers and the Independent Judge across all individual sessions.

\begin{tabular}{|c|c|c|c|c|c|c|c|c|}
\hline Condition & $n^{a}$ & $\begin{array}{l}\text { Total } \\
\text { hits }\end{array}$ & $\begin{array}{c}\text { Hit } \\
\text { rate } \%\end{array}$ & $95 \% \mathrm{CI}$ & $\begin{array}{c}\text { Mean } \\
\text { hits }\end{array}$ & SD & $\begin{array}{l}\text { Most } \\
\text { hits }^{\text {b }}\end{array}$ & Least hits $^{b}$ \\
\hline Judge & 133 & 74 & $56 \%$ & {$[47,62]$} & 10.57 & 1.27 & 12 & 8 \\
\hline $\begin{array}{l}\text { Remote } \\
\text { Viewer }\end{array}$ & 133 & 63 & $47.4 \%$ & {$[39,56]$} & 9 & 2.82 & 13 & 5 \\
\hline
\end{tabular}

Table 2 shows the descriptive statistics for remote viewing sessions, reported as the number of hits, hit percentage, and associated interval estimates for both light and dark conditions for both the remote-viewer and the independent judge. Having a session in the dark appears to produce a marginally higher number of target hits when compared to having a session in the light for both the remote viewers and the independent judge. The independent judge also appears to have been more accurate overall at correctly identifying the target across both light and dark conditions.

Table 2: Descriptive statistics for session hits per Remote Viewer, recorded by Remote Viewers and the Independent Judge for light and dark sessions.

\begin{tabular}{ccccccccccc}
\hline Condition & $\begin{array}{c}\text { Light } \\
\text { hits }\end{array}$ & $\begin{array}{c}\text { Hit } \\
\text { rate\% }\end{array}$ & $95 \%$ CI & $\begin{array}{c}\text { Mean } \\
\text { light }\end{array}$ & SD & $\begin{array}{c}\text { Dark } \\
\text { hits }\end{array}$ & $\begin{array}{c}\text { Hit } \\
\text { rate\% }\end{array}$ & $95 \%$ CI & $\begin{array}{c}\text { Mean } \\
\text { dark }\end{array}$ & SD \\
\hline Judge & 34 & $54 \%$ & {$[41,67]$} & 4.86 & 1.34 & 40 & $57.1 \%$ & {$[45,69]$} & 5.71 & 1.60 \\
$\begin{array}{c}\text { Remote } \\
\text { Viewer }\end{array}$ & 29 & $46 \%$ & {$[33,59]$} & 4.14 & 1.95 & 34 & $48.6 \%$ & {$[36,60]$} & 4.85 & 2.34 \\
\hline
\end{tabular}

To test the first hypothesis, a single sample t-test was used as an approximation of the method initially proposed by Greville (1944) and expanded upon by Burdick and Kelly (1977). The unit of analysis for this test was taken as the average of the independent judge's target-minus-decoy difference ratings for each session.

Due to participants all viewing the same target and decoy pairing in each session, using the session as unit approach conservatively addresses the potential for a stacking effect. Here an overall 
positive mean difference score would indicate an overall tendency to rate the target higher than the decoy, a negative score representing the tendency to rate the decoy higher than the target. While overall a tendency to rate the target higher than the decoy image was shown in the judge's average target-decoy ratings $(M$ diff $=3.76,95 \% \mathrm{CI}[-6.4,13.9], \mathrm{SD}=21.13)$ the findings indicate this tendency is not a significant difference from a score of $0(t(18)=.775, p=.22$ (one-tailed) with a small effect size $(d=.18$, $[-.28, .63])$

In testing the second hypothesis, difference scores between the independent judge and remoteviewers target raw scores were calculated by taking the remote viewers' correspondence raw score from the independent judge's correspondence raw score for each target image. These differences were tested against 0 with a single-sample t-test. The results demonstrated that there was a statistically significant difference between remote viewer and independent judge raw scores attributed to the target image ( $t$ (132) $=4.56, p<.001$ (one-tailed) $M$ diff $=14.21[8.05,20.4])$ with a medium effect size $(d=.40[.21, .57])$. The independent judge was significantly more likely to rate the target image as a higher correspondence to the participant mentation, than the participants themselves.

For testing hypothesis three, the difference values between the independent judge's, minus the remote viewer's correspondence scores for the target image were split across light and dark trial performance. Descriptive statistics are shown in table 3, which shows that while the independent judge rated, on average, the target higher than the remote viewer in both conditions, this was slightly higher for the light sessions.

Table 3: Descriptive statistics for the difference between the Independent Judge's target correspondence raw scores minus the Remote Viewers' self-correspondence target image scores for light and dark sessions.

\begin{tabular}{ccccc}
\hline Condition & $n$ & $M$ & $95 \% \mathrm{Cl}$ & $S D$ \\
\hline Light & 63 & 15.49 & {$[6.29,24.69]$} & 36.53 \\
Dark & 70 & 13.07 & {$[4.55,21.58]$} & 35.69 \\
\hline
\end{tabular}


Despite this, a between measures t-test demonstrated no significant difference $(t(131)=.386, p=$ .35 (one-tailed), $M$ diff $=2.42[-9.98,14.92])$ with a small effect size $(d=.03[-.14, .20])$. This finding suggests that the independent judge was equally likely in both light and dark sessions to more regularly rate the target correspondence higher than the decoy when compared to the remote viewers, with largely overlapping confidence intervals between conditions, as shown in Figure 1.

Figure 1: An error bar graph demonstrating the average Judge-Participant difference values for both light and dark conditions with associated $95 \%$ confidence intervals.

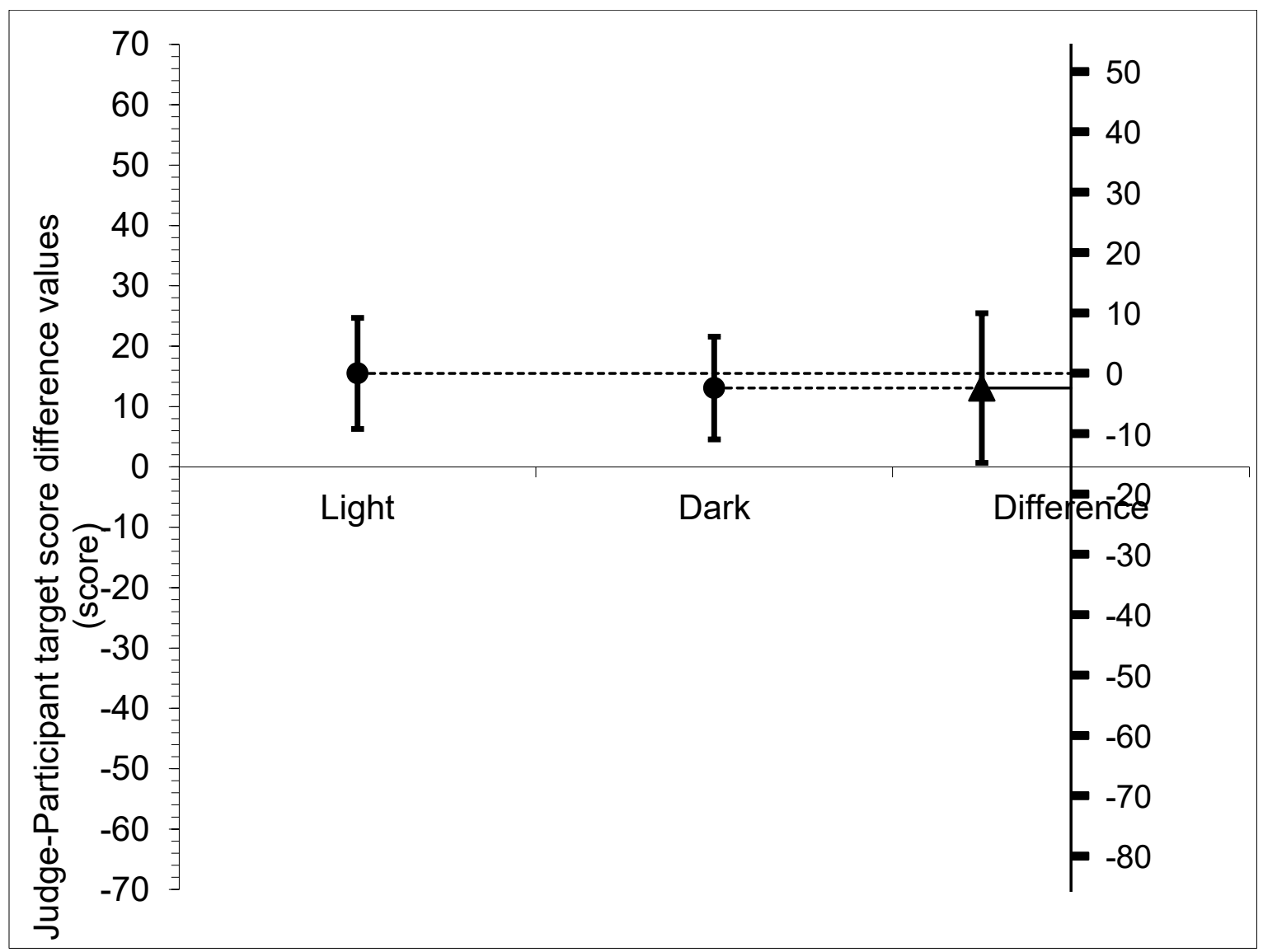

In order to test the fourth hypothesis, a between measures t-test was conducted comparing the independent judge's target-decoy difference values for each remote viewer between light and dark conditions. Descriptive statistics are shown in table 4.

Table 4: Descriptive statistics of the Independent Judge's Target-Decoy difference values for both light and dark conditions.

\begin{tabular}{lllll} 
Condition & $n^{*}$ & $M$ & $95 \% \mathrm{CI}$ & $S D$ \\
\hline
\end{tabular}




\begin{tabular}{lllll} 
Light & 62 & 4.7 & {$[-2.89,12.35]$} & 30.01 \\
Dark & 69 & 5.7 & {$[-1.77,13.33]$} & 31.43 \\
\hline
\end{tabular}

*An extreme score was omitted for both light and dark conditions, for which the decoy score was rated substantially higher than the target score. While not impacting on the results of the inferential analysis their removal provided a more accurate reflection of the data while additionally satisfying the t-tests assumptions.

The results demonstrated no significant difference for the independent judge's target minus decoy difference values between light and dark conditions $(t(129)=.196, p=.845$ (two-tailed), Mdiff $=1.05$ [$9.59,11.71])$ with a negligible effect size $d=.02[-.15, .19])$; this minor difference is demonstrated in

\section{figure 2 .}

Figure 2: An error bar graph demonstrating the average Judge Target-Decoy difference values for both light and dark conditions with associated $95 \%$ confidence intervals.

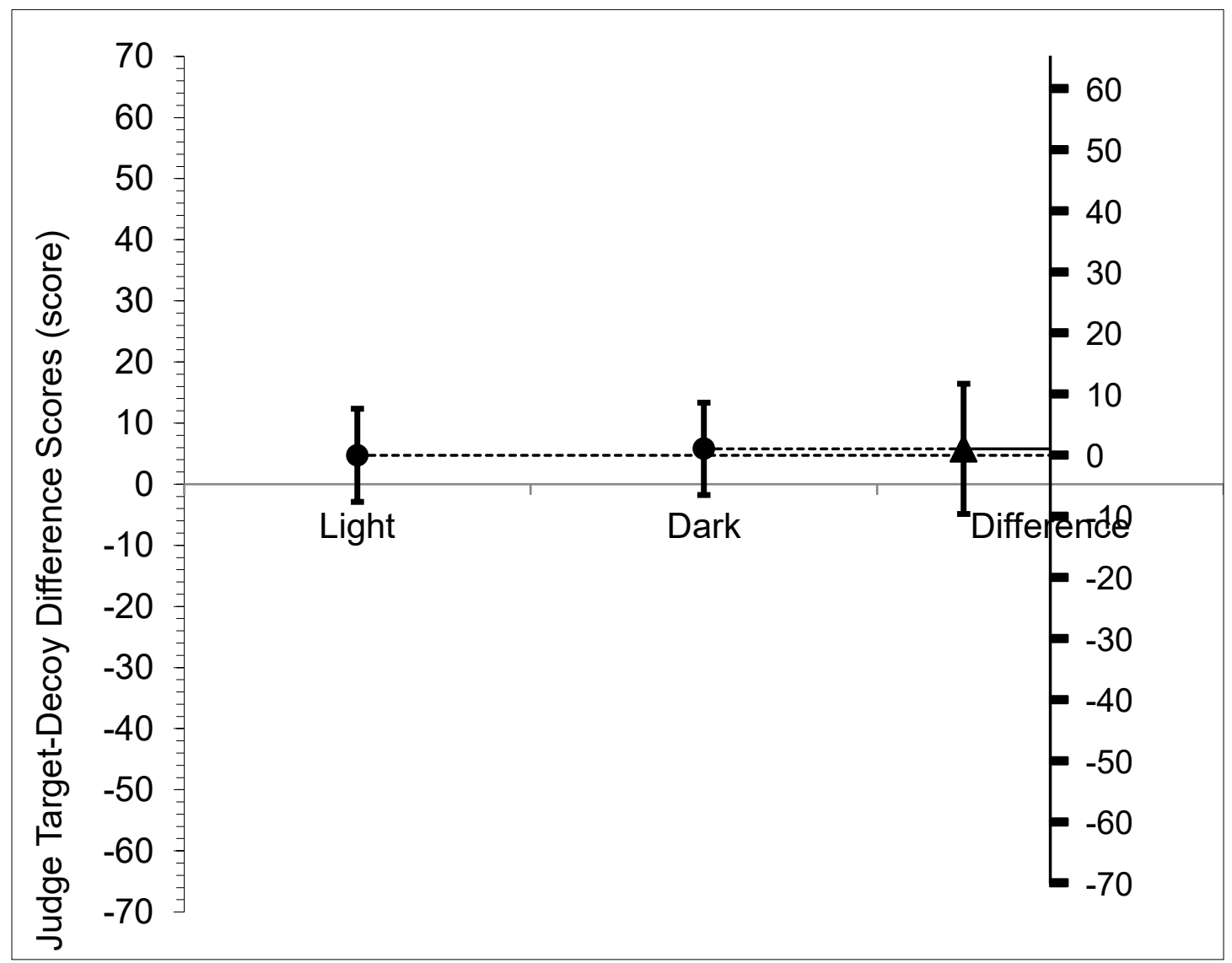

To test the fifth hypothesis, a mixed factorial ANOVA was conducted. Only nine sessions were utilized with the omission of the third dark session; this was to retain temporal similarity between both light and dark sequential trials due to the third light session being discarded from the data set. The 
Sphericity assumption was violated, and thus Greenhouse-Geisser corrected values were used. Overall the factorial ANOVA demonstrated no significant interaction effect for dark/light $\mathrm{x}$ sequential trial $(F(4.1)=$ $\left.2.44, p=.07, \eta_{p}^{2}=.28\right)$

As a posthoc analysis, it was decided to assess the individual participant performance of the most successful participant in the study. The highest scoring individual participant achieved 13 hits out of 19 trials from his judgments. This individual subsequently was also the highest scoring participant for the independent judge scores, receiving 12 out of a possible 19 hits. A binomial test indicated that the proportion of successful hits $(68 \%, 95 \%$ CI $[.46, .84])$ for this individual participant, while near, was not significantly above chance expectation $(p=.08)$.

\section{DISCUSSION}

The original intent of this study was to determine whether ostensible remote viewing functioned better under light or dark conditions. Although the usable data gave the edge to dark conditions, the difference was not statistically significant. In retrospect, the usable data were too small to provide a definitive answer to this question.

When comparing the independent judge's scores against participant scores, hypothesis two was supported with a statistically significant difference, showing the independent judge as more accurate at selecting the correct target. This finding is in line with those presented by Watt (2014) whose dream precognition study demonstrated that participant similarity ratings of targets, while correlated with participants percieved confidence of successful target identification, did not correlate with the significantly above chance hit rate of the independent judges. In this study independent judges performed significantly better, with 11 more hits than the participants own judgements. This suggests that psychological mechanisms may lead to an increased subjective experience of psi in participants which leads them to judge correspondences in a way not condusive to correct target selection. 
This observation has implications for the protocol used in the present study which may lead to extrasensory information from the presentation decoys to participant being incorporated into the content of participant mentations. If independent judges tend to perform better overall there may be little benefit and greater potential for error by using participant judgements. Simply presenting the participant with the target image for feedback and leaving judgements against decoys to the independent judges reduces these issues and also removes the need for more complex steps to be taken to address for stacking effects, during data analysis. An issue highlighted as problematic in studies where a group of participants witness the same target set trial by trial (Kennedy, 1979; Utts, 1995).

\section{Post-Hoc Analysis}

With a dropout rate of over $50 \%$ it was decided to investigating the motivation behind those who elected to drop out; as only seven of the original 20 participants completed 19 usable protocols. Upon inquiry, many of the participants who did not complete all 20 sessions voiced their dissatisfaction with the targets. When queried, a typical response was that participants did not find the target pictures "engaging," "interesting," or "emotionally involving." Many of them attributed their low rate of success to the targets we employed. Schwartz (2007) has written about the importance of choosing RV targets that are endowed with "numinosity" (pp. 114-125). Drawing upon his writings, we defined numinosity as the perceived ability of a target to evoke an emotional state in the participant. We hypothesized that correspondence between the target and participant responses would be significantly correlated with the degree of numinosity of the target.

Charles Honorton developed his target pool when he directed the Psychophysical Laboratory in Princeton, NJ. An inventive experimenter, he is best known for his participation in the Maimonides Medical Center dream experiments (e.g., Krippner, Honorton, \& Ullman, 1971) and for developing the standard Ganzfeld protocol (e.g., Honorton, 1985). At Maimonides, he was part of the team reporting that images evoking strong emotional responses were particularly effective in enhancing psi-related responses, a discovery he carried with him into his later work with his Ganzfeld projects. Specifically, these target 
images involved violence, sexuality, incongruity, novelty, and/or physical movement. Target images, of course, have different meanings for different viewers so the common elements to those cited were either pleasant or unpleasant emotionality or, as we will call it, numinosity. At its most vibrant, numinosity crosses gender, age, culture, and other variables because it is characterized by what might be referred to as coherence.

In a post-hoc analysis (i.e., one not included in the protocol approved by the KPU), there was a significant relationship between numinosity and participants‘ scores; reflecting response-target correspondences. This analysis is the first attempt to evaluate the degree of target numinosity's effect on attempted remote viewing success. Because there are several advantages to what parapsychologists refer to as "free response" targets as opposed to "forced choice" targets (Honorton, 1975), this finding has implications for the selection of target material in future parapsychological research.

Not having access to the research participants or the results of this study, Stephan Schwartz agreed to rate every target for its numinosity on a 100-point scale. "A score of 1 indicated no numinosity, and a score of 100 would indicate full numinosity". Both participant and independent judge scores were used in the analysis.

Honorton's 1975 target pool emphasized incongruity, novelty, and/or physical movement rather than violence and sexuality. When the targets used for this study were evaluated on the 100-point scale for numinosity, the highest total score awarded for any individual target image used in the study was 52.5, with a mean numinosity rating of $22.7,95 \% \mathrm{CI}[17,28.4] \mathrm{SD}=11.86$.

Table 5: Descriptive statistics of numinosity scores organized for light and dark conditions and both Independent Judge and Remote Viewer 'hits' and 'misses' sessions.

\begin{tabular}{ccccccc}
\hline & Light & Dark & $\begin{array}{c}\text { Judge } \\
\text { Hits }\end{array}$ & $\begin{array}{c}\text { Judge } \\
\text { Misses }\end{array}$ & $\begin{array}{c}\text { RV } \\
\text { Hits }\end{array}$ & $\begin{array}{c}\text { RV } \\
\text { Misses }\end{array}$ \\
\hline $\begin{array}{c}\text { Mean } \\
\text { Numinosity }\end{array}$ & 22.22 & 23.12 & 22.5 & 22.87 & 28.61 & 17.37 \\
$95 \%$ CI & & & & & & \\
& {$[14.31$,} & {$[13.33$,} & {$[14.05$,} & {$[13.43$,} & {$[18.04$,} & {$[12.46$,} \\
SD & $30.12]$ & $32.91]$ & $30.94]$ & $32.32]$ & $39.18]$ & $22.28]$ \\
\hline
\end{tabular}

Table 5 shows that the numinosity ratings of target images were evenly distributed across both light and dark conditions with comparable mean numinosity scores, $95 \%$ confidence intervals and 
standard deviation. Independent judge successful (hit) and unsuccessful (miss) sessions overall were comparable regarding the targets' numinosity. There is a noticeable difference however for the mean numinosity ratings between the remote viewer's successful and unsuccessful trials.

For the remote viewing participants, targets in both dark and light conditions appeared to have similar degrees of numinosity associated with hits $(\mathrm{M}=29,95 \% \mathrm{CI}[8,50])$ for dark, and $(\mathrm{M}=28.12$ $[10.6,45.6])$ for light. This similarity remained for missed target numinosity scores $(M=17.25,[8.6$, 25.6], $\mathrm{M}=17.5,[8,27]$ for dark and light respectively.

To investigate the difference in target numinosity between remote viewer hits and misses an independent group t-test was conducted comparing differences in numinosity ratings between sessions in which the participants own mean target-decoy difference values were considered a hit, against those sessions which were considered a miss. A mean difference between groups of $11.24,95 \%$ CI [.12, 22.3] was shown as significant, with the target images of successful 'hit' sessions containing higher numinosity ratings than unsuccessful 'miss' sessions $(\mathrm{t}(11.47)=2.22, p(2$-tailed $)=.048$ with a large effect size $(d=$ $1.02,[.01,1.99])$.

A further independent group t-test was conducted to compare the differences in numinosity rating between sessions for which the independent judge's mean target-decoy difference values were considered a hit, and those sessions considered a miss. A mean difference value of $-.37,95 \%$ CI $[-12.2,11.46]$ between the groups indicated no significant difference in numinosity ratings $(t(17)=-.067, p(2$-tailed $)=$ $.946)$ with a small effect size $(d=.03,[-.87, .93])$ between successful and unsuccessful sessions.

These findings are supportive of the Schwartz (2007) hypothesis, and in line with previous observations by Honorton (1975) and Watt (1989) that targets with emotional content are more likely to be identified as such in remote viewing studies.

Numinosity may be related to the concept of entropy, first observed by Schwartz when participants could accurately identify targets imbued with an element of energetic change (Dossey, 2007). Entropy, characterised by incoherence, and a move towards disorder has received empirical support suggesting it is an information carrying mechanism for successful remote viewing performance (May, 
Spottiswode, \& James, 1994a, 1994b; May, Utts, and Humphrey, 1990; May \& Vilenskaya, 1992). The findings of these studies suggest the higher the entropy gradient of a target, the higher remote viewers' accuracy to correctly identify the target. While movement and change appear essential elements of the nature of remote viewing, emotional factors of numinosity may provide complementary knowledge to understanding what constitutes appropriate remote viewing targets.

An interesting point of note is that while target numinosity was significantly related to successful hits for participants, it appeared to make little difference for the independent judge, who was, in fact, more accurate overall. This suggests that target numinosity may be an essential factor for when participants make their judgements. This may be due to a tendency to focus on the more numinous aspects of their mentation due to the heightened emotional significance. This may also suggest rather than target numinosity being a psi conducive aspect in succesful RV performance, it may reflect a reporting bias where paritcipants are simply more drawn to select stimuli with greater numinous content as the target, seeing greater correspondences with their own experience. Independent judges, unhindered by this may be able to take a more holistic view of participant mentations, leading to the more frequent correct identification of correspondences with the target. This may provide some insight into the difference in accuracy observed between remote viewers and the independent judge. It would be useful in a future study to assess all targets and decoys for their respective numinosity to see if numinosity correlates with rating scores more signiciantly for target clips than for decoys, if so this is supportive of the notion that numinosity is an important element in correct target identification in remote viewing studies. Conversely if a similar relationship is found for decoys, this could reflect a reporting bias.

While the findings of this pilot study are indicative, there are methodological concerns which make their interpretation problematic. The distributed sleeping masks were intended to be worn by participants in both light and dark conditions to act as a control for increased concentration rather than the darkness of the environment. However, this rationale is based on the assumption that darkness of the immediate environment is the crucial factor in facilitating successful remote viewing performance. It may 
be simply a darkened visual field and the limited visual sensory information which accompanies it are the essential ingredients for any improved success within dark conditions rather than the darkness of the environment itself. Indeed, a homogenous visual field has been shown to contribute towards successful psi performance within the Ganzfeld literature (e.g. Bem \& Honorton, 1994; Parker, 2000; Storm, 2006). Thus for participants utilising their sleep masks, effectively both light and dark sessions could be considered dark sessions, potentially violating the independent condition. Furthermore, as some participants found the sleeping mask inhibitive, the decision was made to make the use of these masks optional rather than mandatory, adding additional noise into the data as some participants stopped utilising them entirely while others continued to use them, a consistent approach is highly recommended for future studies.

A further methodological issue is that participants were allowed the use of a small light to write their impressions when in the dark condition, this light was not standardised across participants, and thus some may have used limited sources like a small candle, others a flashlight, leading to additional inconsistencies across trials. While this made it easier for participants to record their impressions, it again risks violating the independent variable of darkness/light. Additionally, if participants were recording their impressions at any point with a pen during the session, they would have to remove the sleeping masks to do so. It is recommended that audio/video recording of participants in future sessions will control for the consistency of the darkness condition across participants, while also allowing participants to merely verbalise their impressions during the trial and then commit their images to paper in the form of an illustration of their impressions after the session period has ended.

Finally, due to the number of trials which occurred throughout the study, and the decision for a binary outcome judgement task, this pilot study was substantially underpowered to find an effect. Despite this point, the findings above can be viewed as indicative and warrant further investigation with a refined protocol and select sample.

Considerable time and effort went into the design and execution of this study. Target selection was carried out in a way that would maximize differences between the actual target and the control target. 
Targets were determined by random selection from a pool constructed by Honorton (1975) that could be evaluated by a binary process based on presence or absence of predesignated information. For example, on September 4, 2014, one of the participants responded, "spikes, Blue, lots of motion, salty, Animal, Feathery texture, Red, pattern [sketch of 3 diagonal parallel lines], voices laughing, crying, loud, closed door, shine reflection, yellow, red [sketch of a long horizontal oval shape around "shine reflection" and a rectangle shape around "red"]. The target image for that session included eight human characters in motion (four of them wearing blue), stacked in what appears to be the bleachers of a ball game, with beer spilling out of cups and hot dogs overflowing with ketchup and mustard. Each character expresses a passionate emotion, e.g., joyful, exhilarated, or depressed, two of the characters appear to be laughing, and two appear to be crying. The central character wears a coat with animal fur around the collar with a feathery texture. A pattern of 3 diagonal parallel lines appears on a flag displaying the word "KILL" backwards as one character waves it in the air. Using Honorton's (1975) binary system, this participant's response would receive a "1" for the presence of: color, activity, mythical characters, animals, human characters, artifacts/implements, food, body parts, architectural objects, and nature features, and a " 0 " for the absence of these content variables resulting in a score of 1101111010 . The target for that night would receive a score of 1110111100 while the decoy target's score would be 1001101101 . It can be seen that the match between the participant's response and the actual target is closer $(60 \%)$ than the match between the response and the control target (50\%). With enough data, a simple statistical test could be made on that participant's collected responses, as well as for the participant group as a whole. However, the participants' responses were returned to Experimenter A in a variety of formats, making Honorton's scoring method unwieldy. As an alternative measure, a 100-point scale was utilized by both participants and the independent judge. The derived scores were easily available for statistical analysis but lacked the more structured and systematic judging approach originally intended, and a clearer response protocol to standardise participant reponses for comparison would be beneficial in a future study. 


\section{Conclusion}

For a proper evaluation of the light/dark hypothesis, a more substantial number of responses needs to be collected, ideally from a small number of carefully selected participants who would dedicate themselves to weekly remote viewing over a period lasting several months. The presentation of the target and decoys to participants also appear to provide little merit to the testing of anomalous cognition with independent judge accuracy significantly higher than those of the participants. In future studies, only the presentation of the target to participants as feedback for each trial is recommended, with all ratings and/or rankings conducted by the independent judge for the target against decoys. This procedure should be accompanied by the standard response protocol if the Honorton target pool is utilized (Honorton, 1975). If not, targets should be evaluated as to their coherence and emotionality (i.e., numinosity) before becoming part of the pool, in order to assess the role of numinosity in sucessful remote viewing more comprehensively. Overall our findings should be considered with caution but warrent further investigation. The accuracy of the independent judges compared to participants, and the potential effect of target numinosity on hits appears to be this study's major contributions to the remote viewing literature.

King Croesus's double-blind experiment of 3,300 years ago has gone through several permutations, but the emphasis on intention remains a prominent concern. Participants' intention underlies reported successful uses of remote viewing in real-world applications. These applications of remote viewing seem to be slowly gaining credence despite criticism and may represent an appropriate way of exploring and applying this elusive phenomenon. Smith and Moddel (2015, p.380) point out that for a practical application to exist, the phenomenon in question must be at least moderately repeatable. Moreover, this is the challenge remote viewing faces in its future development.

\section{Declarations:}

The authors have no competing interests to declare.

This project was supported by the Saybrook University Chair for the Study of Consciousness. 


\section{REFERENCES}

Bem, D. J., \& Honorton, C. (1994). Does psi exist? Replicable evidence for an anomalous process of information transfer. Psychological Bulletin, 115, 4-18.

Burdick, D. S. \& Kelly, E. F. (1977). Statistical methods in parapsychological research. In B.B, Wolman (Ed.). (1977). Handbook of Parapsychology (pp.81-130), New York, NY: Van Nostrand Reinhold.

Dossey, L. (2007). Premonitions, Explore: The Journal of Science and Healing, 4, 83-90.

Gauld, A. (1982). Mediumship and survival: A century of investigations.London, UK: Heinemann.

Greville, T. N. E. (1944). On multiple matching with one variable deck. Annals of Mathematical Statistics, $15,432-434$.

Grim, J. A. (1983). The shaman: Patterns of Siberian and Ojibway healing. Norman, OK: University of Oklahoma Press.

Hallowell, A. I. (1942). The role of conjuring in Saulteaux Society. Publications of the Philadelphia Anthropological Society, 2, Brinton Memorial Series.

Honorton, C. (1975). Objective determination of information rate in psi tasks with pictorial stimuli. Journal of the American Society for Psychical Research, 69, 353-359.

Honorton, C. (1985). Meta-analysis of the psi ganzfeld research: A reply to Hyman. Journal of Parapsychology, 49, 51-91.

Hyman, R. (1995). Evaluation of program on “Anomalous Mental Phenomena.” In M. D. Mumford, A. M. Rose, \& D. A. Goslin (Eds.), An evaluation of remote viewing: Research and applications. (sec. 3, pp. 43-78). Washington DC: The American Institutes for Research.

Inglis, B. (1977). Natural and supernatural. London, UK: Hodder and Stoughton, London.

Keen, M., Ellison, A., \& Fontana, D. (1999) The Scole report: An account of an investigation into the geniuses of a range of physical phenomena associated with a mediumistic group in Norfolk, England. Proceedings of the Society for Psychical Research, 58, 220.

Kennedy, J. E. (1979). Methodological problems in free-response ESP experiments. Journal of the American Society for Psychical Research, 73, 1-16.

Krippner, S., Ullman, M., \& Honorton, C. (1971). A precognitive dream study with a single subject. Journal of the American Society for Psychical Research, 65, 192-201.

Lantz, L. D., Suke, W. L. W., \& May, E. C. (1994). Traget and sender dependencies in anomalous cognition experiments. Journal of Parapsychology, 58, 285-302. 
Laughlin, C. (2011). Communing with the gods. Brisbane, Australia: Daily Grail.

Lyon, W. S. (2012). Spirit talkers: North American medicine powers. Kansas City, KS: Prayer Efficacy Publishing.

May, E. C., Spottiswoode, S. J. P., \& James, C. L. (1994a). Shannon entropy: a possible intrinsic target property. Journal of Parapsychology, 58, 384-401.

May, E. C., Spottiswoode, S. J. P., \& James, C. L. (1994b). Managing the target-pool bandwidth: possible noise reduction for anomalous cognition experiments. Journal of Parapsychology, 58, 303-313.

May, E. C., Utts, J. M., Humphrey, B. S., Luke, W. L. W., Frivold, T. J., \& Trask, V. V. (1990). Advances in remote-viewing analysis. Journal of Parapsychology, 54, 193-228.

May, E. C., \& Vilenskaya, L. (1992). Overview of current parapsychology research in the former Soviet Union. Subtle Energies, 3, 45-67.

Myers, F. W. H. (1880). Greek oracles. In E. Abbott (Ed.), Hellenica: A collection of essays on Greek poetry, philosophy, history and religion (pp. 425-492). London, UK: Rivingtons.

Parker, A. (2000) A review of the Ganzfeld work at gothenburg university. Journal of the Society for Psychical Research, 64, 1-15.

Puthoff, H., \& Targ, R. (1976). A perceptual channel for information transfer over kilometre distances: Historical perspective and recent research. Proceedings of the IEEE, 64, 329-354.

Schwartz, S. A. (2007). Opening to the infinite. Buda, TX: Nemoseen.

Schwartz, S. A. (2014). Through time and space: The evidence for remote viewing. In D. Broderick \& B. Goertzel (Eds.) Evidence for psi: Thirteen empirical research reports. (pp.168-212). Jefferson, $\mathrm{NC}$ : McFarland

Schwartz, S. A. (2016). Why isn't consciousness a fundamental in science? Explore: The journal of science and healing, 12, 403-407.

Schwartz, S. A. (2017, January). Remote viewing. In Psi encyclopedia. Retrieved from https://psiencyclopedia.spr.ac.uk/articles/remote-viewing

Smith, P.H., \& Moddel, G. (2015). Applied psi. In E. Cardena, J. Palmer, \& D. MarcussonClavertz (Eds.), Parapsychology: A handbook for the $21^{\text {st }}$ century (pp. 380-388). Jefferson, NC: McFarland.

Storm, L. (2006). Meta-Analysis in parapsychology: I. The Ganzfeld domain. Australian Journal of Parapsychology, 6, 35-53.

Targ, R., \& Puthoff, H. (2005). Mind-reach: Scientists look at psychic abilities. Newburyport, MA: Hampton Roads.

Tressoldi, P. E. (2011). Extraordinary claims require extraordinary evidence: The case of non-local perception, a classical and Bayesian review of evidences. Frontiers in Psychology, 117 (2), 1-4.

Utts, J. (1995). An assessment of the evidence for psychic functioning. In M. D. 
Mumford, A. M. Rose, \& D. A. Goslin (Eds.), An evaluation of remote viewing: Research and applications (sec. 3-2). Washington DC: The American Institutes for Research.

Watt, C. (1989). Characteristics of successful free-response targets: Theoretical considerations. In L. A. Henkel \& R. E. Berger (Eds.) Research in Parapsychology 1988 (pp. 95-99). Metuchen, NJ: Scarecrow Press

Watt, C. (2014). Precognitive dreaming: investigating anomalous cognition and psychological factors. Journal of Parapsychology, 78, 115-125. 